\title{
Application of Sine-Cosine Optimization Algorithm for Minimization of Transmission Loss
}

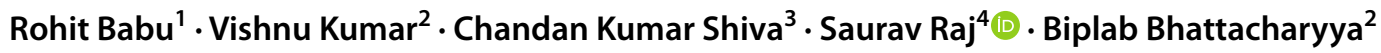

Received: 12 March 2021 / Accepted: 26 January 2022 / Published online: 15 February 2022

(c) The Author(s), under exclusive licence to Springer Nature Singapore Pte Ltd. 2022

\begin{abstract}
On recent trends, the reactive power planning problem has received a considerable amount of attention for the allocation of reactive power resources, both static and dynamic such as switchable capacitors and/or reactors, as well as Var compensators, respectively. The reactive power has a significant impact on voltage profile, stability and transmission loss in power systems. In recent years, voltage instability is recognized as a major threat to system operation due to a shortage of reactive power in an interconnected transmission line. These voltage conditions could be analyzed and improved by proper coordination of reactive sources/sink. In this article, the minimization of transmission loss and operating cost is performed by using the sine cosine algorithm (SCA) and quasi-oppositional based sine cosine algorithm (QOSCA) technique for standard IEEE 14 and IEEE 30 and IEEE 57 bus test system of the connected power network. The proposed QOSCA reduces the transmission losses by $6.38 \%$ in IEEE 14, 4.56\% in IEEE 30 and $12.77 \%$ in IEEE 57 bus system. The results obtained by QOSCA and SCA are compared with other popular techniques recently reported in recent state-of-literature. It is observed that QOSCA yields better results in terms of reducing transmission loss, operating costs, and improving the voltage profile of each bus.
\end{abstract}

Keywords Reactive Power Planning · Active Power Loss · Sine Cosine Algorithm · Quasi-oppositional method

Saurav Raj

sauravsonusahu@gmail.com

Rohit Babu

rohit.babu@lendi.org

Vishnu Kumar

vishnum711@gmail.com

Chandan Kumar Shiva

chandankumarshiva@gmail.com

Biplab Bhattacharyya

biplab@iitism.ac.in

1 Department of Electrical \& Electronics Engineering, Lendi Institute of Engineering and Technology, Jonnada, Andhra Pradesh- 535005, India

2 Department of Electrical Engineering, Indian Institute of Technology Indian School of Mines, Dhanbad 826004, India

3 Department of Electrical and Electronics Engineering, S R Engineering College, Ananthsagar, Hasanparthy, Warangal, Telangana, India

4 Department of Electrical Engineering, Institute of Chemical Technology Mumbai, Marathwada Campus , Jalna 431203, India

\section{Introduction}

Generally, reactive power planning (RPP) is defined as an allocation of additional reactive power sources that should be installed in the network for a predefined horizon of planning at minimum cost while satisfying equality and inequality constraints. The optimal implementation of new reactive sources can be deployed according to specific indices, satisfying the objectives of the problem. However, reactive power sources are needed for increasing the capacity of transmission lines, correction of power factor, reduction of losses, and voltage profile improvement. RPP is an essential issue, especially in modern power systems. It can also be applied without supporting reactive power in some transmission networks.

Further, load bus voltages magnitudes may differ from their permissible limits, which may cause not only unacceptable power quality but the increment in loss of real power. In such cases, additions of the transmission line in an existing network is required which may not be economical. However, the RPP problem is combinatorial and is required to be solved via mixed-integer nonlinear programming. The objective of the RPP problem is to determine 'where,' 'what,' 
'when' and 'how much' new devices are needed to an existing network to make its process reasonable for a pre-defined horizon of planning at minimum total costs.

Authors have implemented simulated annealing techniques in $[1,2]$ to determine locations to install capacitors and optimal control settings of these reactive sources. A convex chance-constrained model for long term investment planning and operation is discussed in [3]. The solution for reactive power planning is determined for the Iranian power grid using a Genetic algorithm in [4]. The mathematical model for solving transmission expansion and reactive power planning problems is discussed in [5]. An adaptive differential evolution procedure is presented in [6] to solve optimal reactive power dispatch problem, and authors have adopted an adaptive penalty factor to alleviate the effects of the dependent variable violation. A parallel hurricane optimization algorithm is proposed in [7] for solving the economic load dispatch problem. A novel application of Tree-shed algorithm to solve optimal power flow problem in a large-scale electric power system are described in [8]. Energy storage in grid-connected microgrid under uncertain real-time prices are discussed in [9]. The chance-constrained optimization algorithm is studied in [10] for the risk-assessment approach for the solution of the RPP problem.

Transmission expansion and reactive power problems are formulated as mixed-integer linear programming in [11]. The expected violation index for security-constrained RPP problems is discussed in [12]. An RPP optimization model is discussed in [13] by an enhanced simulated annealing (SA) algorithm taking advantage of the modified Gray code. A seeker optimization algorithm is proposed in [14] for the solution of reactive power dispatch. A solution of reactive power planning problem is determined by loss sensitivity approach in [15], Teaching learning-based optimization algorithm in [16], hybrid harris hawk particle swarm optimizer in [17] and hybrid forms of particle swarm optimization (PSO) algorithm, oppositional based grey wolf optimization (OGWO) algorithm in [18]. Management of reactive power is proposed in [19] based on fuzzy. RPP problem, along with the voltage stability margin, is evaluated by the differential evolution technique in [20]. Sine Cosine optimization algorithm is introduced in [21]. The concept of oppositional based learning was introduced by Tizhoosh [22].

Transmission expansion planning model along with second-order cone programming (SOCP), has been proposed in [23] for high penetration of wind energy. Optimal reconfiguring of the Algerian distribution electrical system with FACTS devices has been proposed in [24] using a fractal search algorithm. The ameliorated Harris hawk optimizer has been proposed in [25] for the solution of Optimal Reactive Power planning problems. A two-stage strategy like dynamic multiyear transmission expansion planning and transmission expansion planning problem has been developed in [26]. Article [27] proposes a planning strategy based on soft computing techniques to determine the system energy loss and economic benefit for the standard test system. The proposed work in [28] considered variants of PSO to improve the reduction in the total cost of energy loss and real power loss for standard New England 39 bus system. Weak bus detection methods are discussed in [29] and constrained VAr planning using penalty successive conic programming in [30]. A summary of optimization strategies for reactive power planning problems is summarized with its merits and demerits of the various approaches as shown in Table 1.

The unique visage of the sine-cosine algorithm is the ability not to get trapped in local minima, more comfortable to implement and gives the same result even after many trails. The silent features of the proposed QOSCA approach for RPP can be summarized as below:

a) Efficiently allocating reactive power sources during the planning and consequently decreasing the operating cost.

b) Improvement of voltage profile is also obtained on each bus.

c) Sine Cosine optimization algorithm appears to be the best method for minimizing the transmission loss at minimum operating cost.

d) The proposed Quasi-oppositional based Sine Cosine algorithm alleviates the drawbacks of the recently developed optimization algorithms.

e) Applications of standard IEEE 14, IEEE 30 and IEEE 57 bus test systems are carried. Therefore, the capability of the Sine Cosine algorithm is achieved.

In this article, Quasi-oppositional based Sine-Cosine algorithm has been proposed to solve the RPP problems for minimization of the transmission power loss and system operating cost while satisfying all the constraints. The main advantage of the SCA technique is that it does not get trapped in local minima. The proposed QOSCA optimization technique is applied to standard IEEE 14, IEEE 30 and IEEE 57 bus systems of a connected power network. The obtained results are compared with different techniques in the published articles, which states the capability and feasibility of the proposed SCA for the solution of RPP problems.

The organization of the rest of the paper is done in the following way. Section 2 includes a mathematical problem formulation including applicable constraints pertaining to the RPP problem. The description and application of QOSSA and SSA techniques are established in Sect. 3. Section 4 discusses simulation results for both the test system followed by concluding remarks in Sect. 5. 


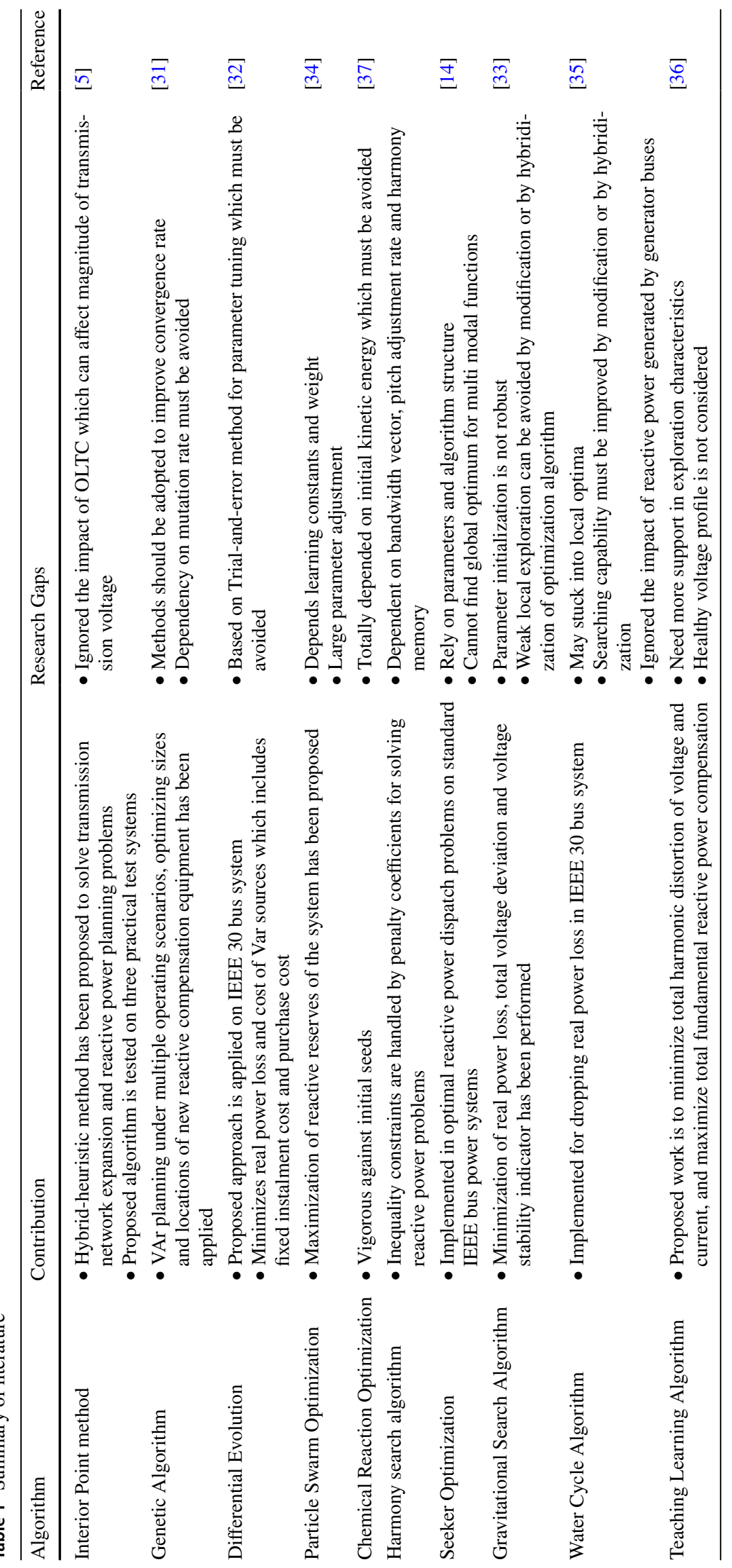




\section{Problem Formulation}

The prime importance of RPP is to minimize the active power losses and operating costs of the system. This planning is done through a proper organization and control of variables, which are transformer tap setting arrangements, shunt capacitors and generation of reactive power. Different advanced techniques have been developed to reduce the line losses in order to achieve an efficient and economic system while keeping bus voltage within an acceptable limit. The operating cost is minimized by cost due to reduced energy losses and the proper installation of capacitors at weak buses. The following objective functions and constraints are considered for the problem of RPP:

The active power loss in the line can be calculated as:

Minimize, $F_{1}=\min \left(P_{\text {Loss }}\right)=\sum_{k=1}^{N_{L}} g_{k}\left(V_{i}^{2}+V_{j}^{2}-2 V_{i} V_{j} \cos \left(\delta_{i}-\delta_{j}\right)\right)$

where, $\mathrm{P}_{\text {loss }}$ represents active power loss, $\mathrm{g}_{\mathrm{k}}$ is the conductance of the $\mathrm{k}^{\text {th }}$ branch connected between $\mathrm{i}^{\text {th }}$ and $\mathrm{j}^{\text {th }}$ branch. $\mathrm{N}_{\mathrm{L}}$, is the number of transmission lines, $\mathrm{V}_{\mathrm{i}}$ and $\mathrm{V}_{\mathrm{j}}$ is the voltage magnitude at the ith and jth bus respectively. $\left(\delta_{i}-\delta_{j}\right)$ is the voltage angle difference between $\mathrm{i}^{\text {th }}$ and $\mathrm{j}^{\text {th }}$ bus.

The operating cost of the system can be evaluated as:

The cost due to energy losses and the cost of the shunt capacitors is minimized to have an economical operation.

Cost of installing capacitor $=1000 \$$,

Energy rate $=0.06 \$ / \mathrm{kWh}$,

capacitor cost $/ \mathrm{kVar}=3 \$$

Energyrate $=0.06 \times 10000 \times 8760$

$$
\begin{aligned}
\text { Total operating } \cos t \text { of system } & =P_{\text {Loss }} \times \text { Energy } \operatorname{Cos} t \\
& + \text { Cost of shunt capacitor }
\end{aligned}
$$

It is a conflicting objective to minimize both real power loss and maintaining voltage within permissible limits simultaneously. Hence, it is done by proper controlling of different control variables represented by a vector $\mathrm{U}$, consisting of reactive power generator $Q_{g}^{i}$, shunt capacitor value $Q_{c}^{i}$ and tap setting $T_{t}^{i}$ as:

$U=\left[Q_{g}^{1}, \ldots, Q_{g}^{n p v}, Q_{c}^{1}, \ldots, Q_{c}^{n c}, T_{t}^{1}, \ldots, T_{t}^{n t}\right]$

where, $n p v$ is the total number of generator buses, $n c$ is the total number of shunt capacitors and $n t$ is the total number of tap changing transformers.

A dependent variable $\mathrm{X}$ associated with active power loss consisting of slack bus power $P_{g}$, the voltage of the load bus $V_{l}^{i}$, reactive power of a generator $Q_{g}^{i}$ and transmission line loads can be represented as:
$X=\left[P_{g}, V_{l}^{1}, \ldots, V_{l}^{n p q}, Q_{g}^{1}, \ldots, Q_{g}^{n p v}\right]$

where, $n p q$ is the number of load buses, $n p v$ is the number of PV buses.

Equality constraints:

These are load flow equations, active and reactive power constraints for any $\mathrm{i}^{\text {th }}$ bus.

$P_{g}^{i}-P_{d}^{i}=\sum_{j=1}^{N_{b}} V_{i} V_{j}\left(G_{i j} \cos \theta_{i j}+B_{i j} \sin \theta_{i j}\right)$

$Q_{g}^{i}-Q_{d}^{i}=\sum_{j=1}^{N_{b}} V_{i} V_{j}\left(G_{i j} \sin \theta_{i j}-B_{i j} \cos \theta_{i j}\right)$

where, $N_{b}$ is the number of buses. $P_{g}^{i}$ and $P_{d}^{i}$ are the active power generation and demand of the $\mathrm{i}^{\text {th }}$ bus whereas, $Q_{g}^{i}$ and $Q_{d}^{i}$ are the reactive power generation and demand at the $\mathrm{i}^{\text {th }}$ bus. $G_{i j}$ and $B_{i j}$ are the real and the imaginary admittance values respectively.

Inequality constraints-Generator constraints:

$Q_{g_{\text {min }}}^{i} \leq Q_{g}^{i} \leq Q_{g_{\text {max }}}^{i}, \mathrm{i}=1,2,3, \ldots, N_{g}$

$V_{g_{\min }}^{i} \leq V_{g}^{i} \leq V_{g_{\max }}^{i}, \mathrm{i}=1,2,3, \ldots, N_{g}$

where, $N_{g}$ is the number of generator buses, $Q_{g_{\min }}^{i}$ and $Q_{g_{\max }}^{i}$ are minimum and maximum reactive power generation, $V_{g_{\text {min }}}^{i}$ and $V_{g_{\max }}^{i}$ are the upper and lower limits of all the particular generator buses.

Limitation on reactive power injections:

$Q_{c_{\min }}^{i} \leq Q_{c}^{i} \leq Q_{c_{\max }^{i}}^{i}$

where, $Q_{c_{\min }}^{i}$ and $Q_{c_{\max }}^{i}$ are the reactive power injection limit by the shunt capacitors.

Transformer tap setting constraints:

$T_{\min }^{k} \leq T_{p}^{k} \leq T_{\max }^{k}$

$\mathrm{k}=1,2,3, \ldots$, ntap.where, ntap is the number of taps changing transformer branches.

Line flow limits:

$S_{l}^{i} \leq S_{l_{\max }^{i}}^{i}$

where, $S_{l_{\max }}^{i}$ is the maximum load on the $\mathrm{i}^{\text {th }}$ line.

Limitation on bus voltage:

$V_{l_{\min }}^{i} \leq V_{l}^{i} \leq V_{l_{\max }^{i}}^{i}$

$\mathrm{i}=1,2,3 \ldots \ldots \ldots \mathrm{nb}$.

$V_{l_{\min }}^{i}$ and $V_{l_{\max }}^{i}$ are the minimum and maximum voltage limits, $\mathrm{nb}$ is the total number of load buses. 


\section{Sine Cosine Optimization Algorithm}

Sine cosine optimization algorithm is a population-based optimization algorithm that is based on the mathematical function of Sine \& Cosine. This algorithm has been proposed by Mirjalili [21] in 2016. In the present work, SCA is used as the optimization algorithm for reactive power planning. Initially, SCA creates multiple random solutions and these solutions then fluctuate towards or outwards the best solution on sine cosine functions given by the mathematical model of SCA.

The optimization process is divided into two-phase that is, exploration and exploitation. In exploration, promising regions of search space are obtained, as the optimization algorithm combines with the random solutions in the set of solutions precipitously with a high rate of randomness. In exploitation, there is a gradual change in random solutions, and random variations are less as compared to exploration. Mathematically, exploration and exploitation of updating positions can be expressed as:

$X_{t+1}^{i}=X_{t}^{i}+r_{0} \times \sin \left(r_{1}\right) \times\left|r_{2} P_{t}^{i}-X_{t}^{i}\right|$

$X_{t+1}^{i}=X_{t}^{i}+r_{0} \times \cos \left(r_{1}\right) \times\left|r_{2} P_{t}^{i}-X_{t}^{i}\right|$

The two equations can be used depending on the factor $r_{3}$ as:

$X_{t+1}^{i}=\left\{\begin{array}{l}X_{t}^{i}+r_{0} \sin \left(r_{1}\right) \times\left|r_{2} P_{t}^{i}-X_{t}^{i}\right|, r_{3}<0.5 \\ X_{t}^{i}+r_{0} \cos \left(r_{1}\right) \times\left|r_{2} P_{t}^{i}-X_{t}^{i}\right|, r_{3} \geq 0.5\end{array}\right.$

where, $X_{t}^{i}$ is the position of the present solution in $\mathrm{i}^{\text {th }}$ dimension at $\mathrm{i}^{\mathrm{th}}{ }^{t}$ iteration. $r_{0}, r_{1}, r_{2}, r_{3}$ are random numbers. $P$ is the position of the destination point in $\mathrm{i}^{\text {th }}$ dimension, $\|$ is the absolute value. In order to achieve a balance between the exploration and exploitation phase, the dynamic fine-tune of $r_{0}$ during the search process is carried out using Eq. (16).

$r_{0}=a-(a \times$ iter $) /$ itermax

where, $a$ is a constant, iter is the current iteration and itermax is the maximum number of iterations.

$r_{1} \in[0,2 \pi]$

$r_{2} \in[0, a]$

$r_{3} \in[0,1]$

$r_{0}$ determines the movement direction, either in the region between the solution and the destination or outside it. $r_{1}$ determines the depth of movement, towards or outwards the destination.$r_{2}$ brings a random weight for the destination in order to stochastically emphasize $\left(r_{2}>1\right)$ or de-emphasize $\left(r_{2}<1\right)$ the effect of destination in defining the distance. $r_{2}$ equally shift between sine and cosine components.
Opposition-based learning

Opposition based learning (OBL) was first instituted by Tizhoosh [22], which can potentially accelerate the convergence of optimization techniques and has proved immensely beneficial for computational intelligence. Any evolutionary or swarm optimization methods begin with the initial solution (initial population generation) which improves and updates to reach some optimal solution finally. Termination is based on setting up predefined criteria and computation time is a critical component to evaluate the robustness and effectiveness of a given algorithm depends on the distance between the initial guess and optimal solution. Fitness evaluation or the chances to arrive at the optimal solution enhances by checking the opposite guesses. This forms the basic framework of opposition-based learning.

So, the fitter one whether the guess or different guess, can be applied to the initial solution and subsequently to each solution in the current population to intensify convergence. Opposition based learning primarily benefits by increasing the probability of even visiting the unproductive regions. Also, it has been established through research that the different solution has a higher possibility to inch towards global optima as compared to a random solution [23]. Mathematically, OBL can be described as follows:

\section{Quasi-oppositional SCA}

In this section, the author proposes a hybrid optimization technique of QOSCA by introducing an opposition-based learning concept in the SCA technique. All swarm optimization techniques commence with some initial solution or initialization, upgrading by fitness evaluation and terminating at an optimal solution.

In the proposed novel hybrid technique of QOSCA, SCA is considered as the parent algorithm and Quasi-oppositional optimization is embedded in SCA to accelerate the speed of convergence. QOSCA engages opposite points for initializing the population and generation jumping and incorporates fitter candidate solutions from the start of the optimization. The mathematical model of a quasi-opposite point is discussed below:

(i). Opposite point

Let $X_{j}^{o}$ be any control variable $\in\left[X^{\max }, X^{\min }\right]$, then any opposition variable can be obtained as

$O X_{j}=X_{j}^{\max }+X_{j}^{\min }-X_{j}^{0}$

In this work, the maximum and minimum opposition variables are reactive power generation limits, transformer tap settings and shunt compensation limits as described below: 


$$
\begin{aligned}
& X_{j}^{\max }=\left[\begin{array}{lllllllll}
Q_{G 1}^{\max } & \ldots & Q_{G j}^{\max } & T_{1}^{\max } & \ldots & T_{j}^{\max } & Q C_{1}^{\max } & \ldots & Q C_{j}^{\max }
\end{array}\right] \\
& X_{j}^{\min }=\left[\begin{array}{llllllllll}
Q_{G 1}^{\min } & \ldots & Q_{G j}^{\min } & T_{1}^{\min } & \ldots & T_{j}^{\min } & Q C_{1}^{\min } & \ldots & Q C_{j}^{\min }
\end{array}\right]
\end{aligned}
$$

Therefore, the opposition matrix and quasi-opposition matrix are given by Eq. (18) and (19) respectively.

$O X=\left[\begin{array}{cll}X_{11}^{\max }+X_{11}^{\min }-X_{11}^{0} & \ldots & X_{1 j}^{\max }+X_{1 j}^{\min }-X_{1 j}^{0} \\ \ldots & \ldots & \ldots \\ X_{i 1}^{\max }+X_{i 1}^{\min }-X_{i 1}^{0} & \ldots & X_{i j}^{\max }+X_{i j}^{\min }-X_{i j}^{0}\end{array}\right]$

where, $i=$ Number of population and $j=$ Number of variables.

$Q O X=\left[\begin{array}{ccc}Q O X_{11} & \ldots & Q O X_{1 j} \\ \ldots & \ldots & \ldots \\ Q O X_{i 1} & \ldots & Q O X_{i j}\end{array}\right]$

The convergence speed is accelerated by selecting a quasiopposite population matrix as the initial population. The generation of the next population is guided by jumping rate and this is incorporated in SCA optimization to increase the effectiveness and robustness of the algorithm. The computational steps followed in the new technique of QOSCA are given as follows:

Algorithmic perspective for the proposed work using Sine-Cosine optimization algorithm is given below:

Step 1. Define test system data.

Step 2. Define maximum iteration and agents.

Step 3. Set the upper and lower boundary limits of control variables like reactive power generation, transformer tap settings.

Step 4. Generate population matrix.

Step 5. Check the inequality constraint limits for the position of population matrix, if they are satisfied, then go to the next step; otherwise again generate population matrix and until all the inequality constraints of Eq. (7) to Eq. (12) are satisfied.
Table 3 Boundary of transformer tap setting and shunt capacitor

\begin{tabular}{llll}
\hline Variables & Test system & $\begin{array}{l}\text { Mini- } \\
\text { mum } \\
\text { Value } \\
\text { (p.u) }\end{array}$ & $\begin{array}{l}\text { Maximum } \\
\text { Value (p.u) }\end{array}$ \\
\hline Shunt capacitor [19] & IEEE 14 & 0.0 & 0.15 \\
& IEEE 30 & 0.0 & 0.15 \\
& IEEE 57 & 0.0 & 0.30 \\
Transformer tap setting [19] & IEEE 14 & 0.9 & 1.0 \\
& IEEE 30 & 0.9 & 1.0 \\
& IEEE 57 & 0.9 & 1.0 \\
\hline
\end{tabular}

Step 6. Initialize $r_{1}, r_{2}$ and $r_{3}$. Then by using the Eq. (16), determine moment direction for the current iteration.

Step 7. Update the position of search agents by exploration and exploitation phase from Eq. (13) to Eq. (15).

Step 8. Update the test system data with a new population string.

Step 9. Y-bus is formed.

Step 10. Newton Raphson program is executed and the objective function is determined using Eq. (1) and Eq. (3) while satisfying equality and inequality constraints of Eq. (5) and Eq. (12).

Step 11. Repeat Step-(6) to step-(10) for all the search agents. Now compare the optimum result value with all the search agent solutions. Store the minimum value of the optimum result and the corresponding position of search agents.

Step 12. Set the iteration number equal to 1 .

Step 13. Repeat step 4 to step 11. Then increase the current iteration by 1 .

Step 14. Now repeat step 4 to step 11 until the maximum number of iteration it has reached.

Step 15. If the current iteration is equal to the maximum number of iteration, then terminate the iterative process. Save as the best solution to optimization problems.
Table 2 Boundary of reactive power generation at the

\begin{tabular}{|c|c|c|c|c|c|c|c|}
\hline \multicolumn{8}{|l|}{ IEEE 14 BUS SYSTEM } \\
\hline Generator Bus & 2 & 3 & & 6 & & & 8 \\
\hline Minimum Value (in p.u) [19] & -0.40 & 0.0 & & -0.06 & & & -0.06 \\
\hline Maximum Value (in p.u) [19] & 0.50 & 0.40 & & 0.24 & & & 0.24 \\
\hline \multicolumn{8}{|l|}{ IEEE 30 BUS SYSTEM } \\
\hline Generator Bus & 2 & 5 & 8 & & & 11 & 13 \\
\hline Minimum Value (in p.u) [19] & -0.20 & -0.15 & -0.15 & & & -0.10 & -0.15 \\
\hline Maximum Value (in p.u) [19] & 0.60 & 0.625 & 0.50 & & & 0.40 & 0.45 \\
\hline \multicolumn{8}{|l|}{ IEEE 57 BUS SYSTEM } \\
\hline Generator Bus & 2 & 3 & 6 & & 8 & 9 & 12 \\
\hline Minimum Value (in p.u) & -0.17 & -0.10 & -0.08 & & -1.40 & -0.03 & -0.50 \\
\hline Maximum Value (in p.u) & 0.50 & 0.60 & 0.25 & & 2.0 & 0.09 & 1.55 \\
\hline
\end{tabular}
generator bus 


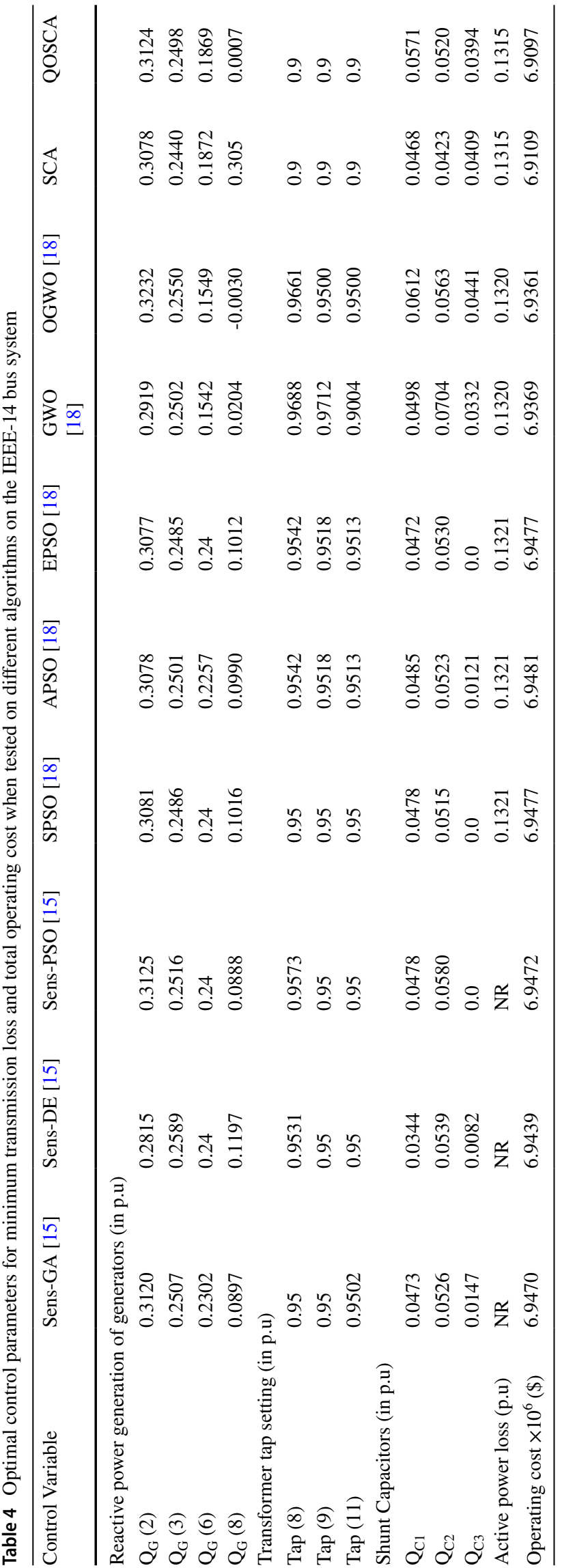

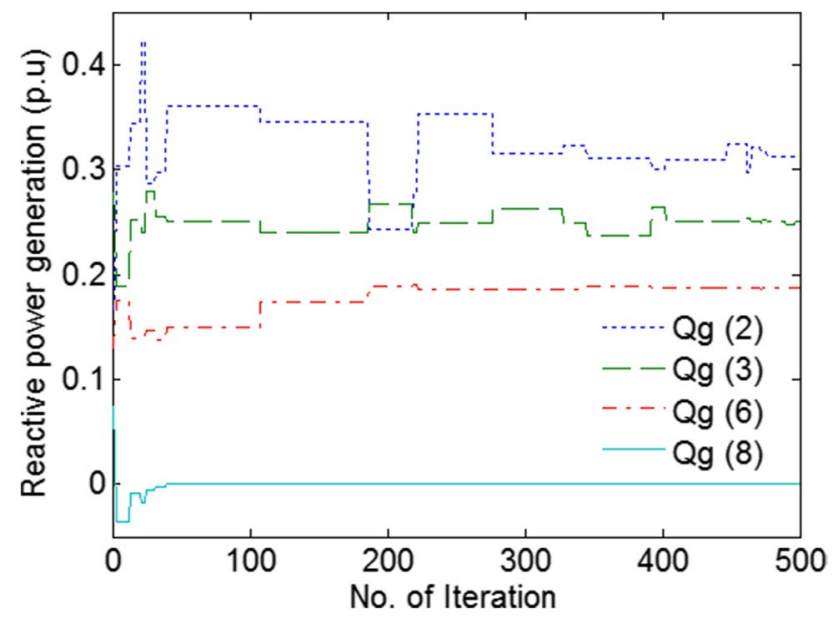

Fig. 1 Variation of Reactive power generation profile

\section{Result and discussion}

In order to demonstrate the applicability and validity of the proposed Quasi-oppositional based Sine Cosine optimization and Sine Cosine optimization algorithm technique for the solution of reactive power planning, standard IEEE 14, IEEE 30 and IEEE 57 bus test system has been considered for the testing purpose. Boundary limits of control variables of these test systems are shown in Tables 2 and 3. In order to validate the proposed optimization algorithm, it has been made to run for 500 iterations and 40 number of search agents in each of the given test systems.

In IEEE 14 bus test system, there are four generator buses with interconnected 20 transmission lines. Three branches are equipped with tap changing transformer and one shunt capacitor. Initially, active power loss without reactive power planning is $13.99 \mathrm{MW}$ and its operating cost is $7.3531 \times 10^{6} \$$. The search space of this test system has 10 dimension that includes four reactive power generators, three transformer tap settings

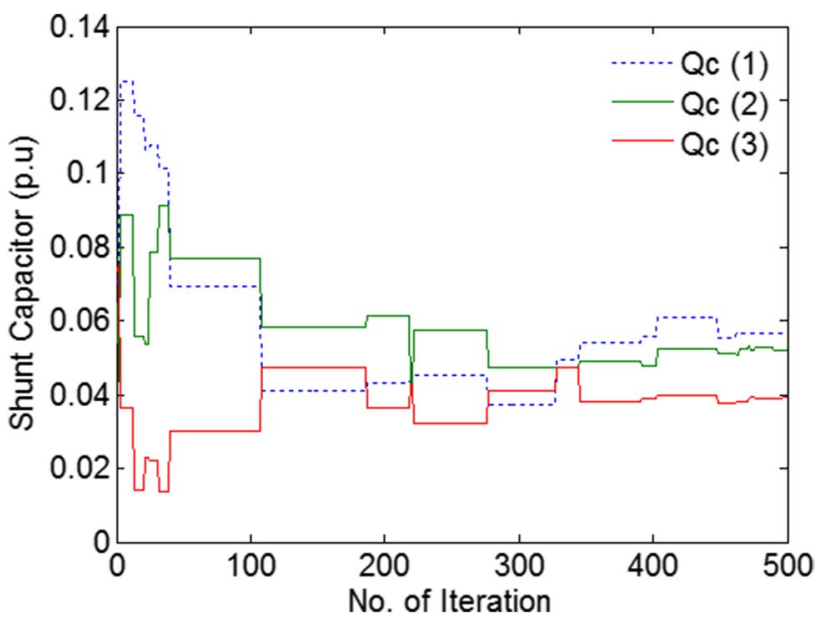

Fig. 2 Variation of Shunt capacitors for $\mathrm{Q}_{\mathrm{c}}(1), \mathrm{Q}_{\mathrm{c}}(2)$ and $\mathrm{Q}_{\mathrm{c}}(3)$ 


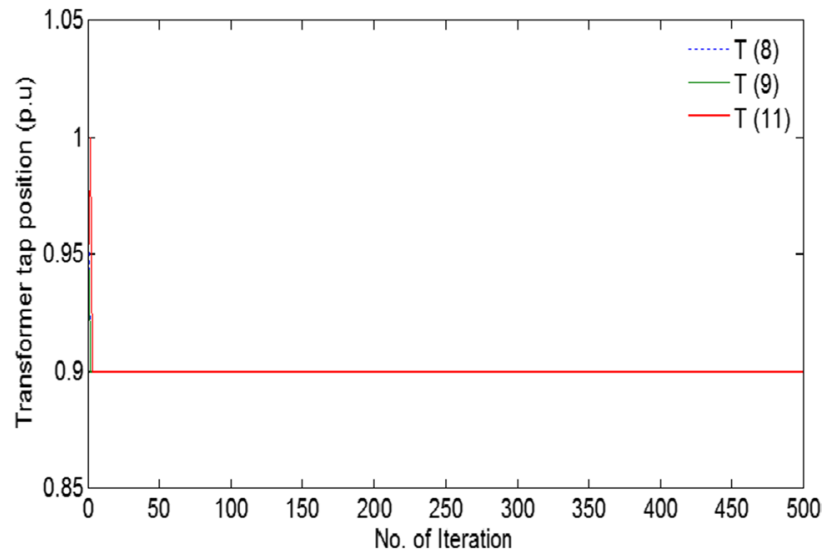

Fig. 3 Variation of Transformer Tap settings for T (6-9), T (6-10) and $\mathrm{T}(28-27)$

and shunt capacitors are placed at weak buses, where a large amount of reactive power is present. Table 4 represents the control parameters obtained by the proposed SCA. The results of proposed SCA are also compared with other optimization algorithm like Sens-GA [15], Sens-DE [15], Sens-PSO [15], SPSO [18], APSO [18], EPSO [18], GWO [19] and OGWO [19]. The variation of reactive power generation profile is shown in Fig. 1. The variation of shunt capacitors for Qc (1), Qc (2) and Qc (3) is shown in Fig. 2. With the application of the proposed approach, total active power loss is reduced to $0.1315 \mathrm{p}$.u and system operating cost is also reduced to $6.9234 \times 10^{6} \$$. Figure 3 depicts the reactive power generation variation at the generator buses during every iteration. Figures 4 and 5 displays the transformer tap setting condition and reactive power variation of shunt capacitors respectively. The minimization of the active power loss curve is shown in Fig. 6 and the convergence characteristics curve of the system operating cost is shown in Fig. 7. The convergence curve

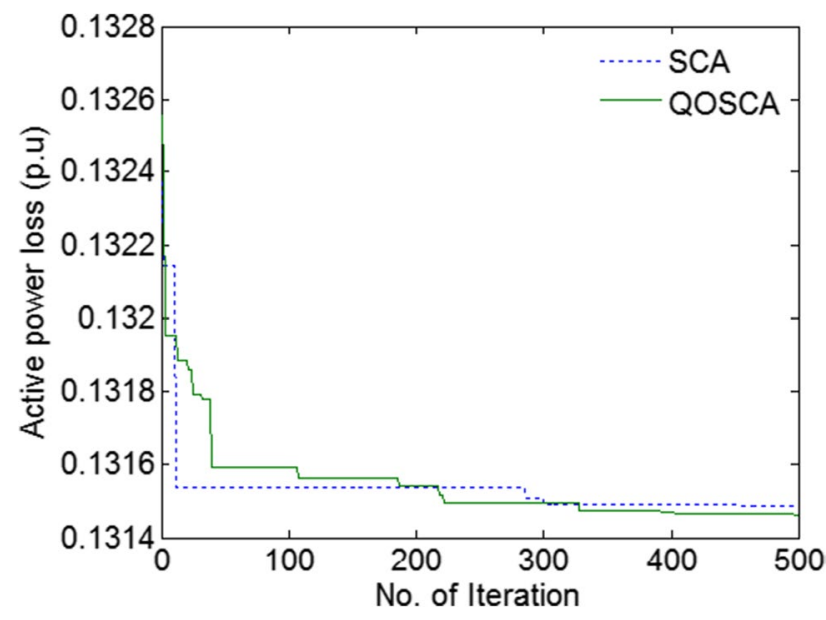

Fig. 4 Convergence curve of active power loss using SCA and QOSCA technique

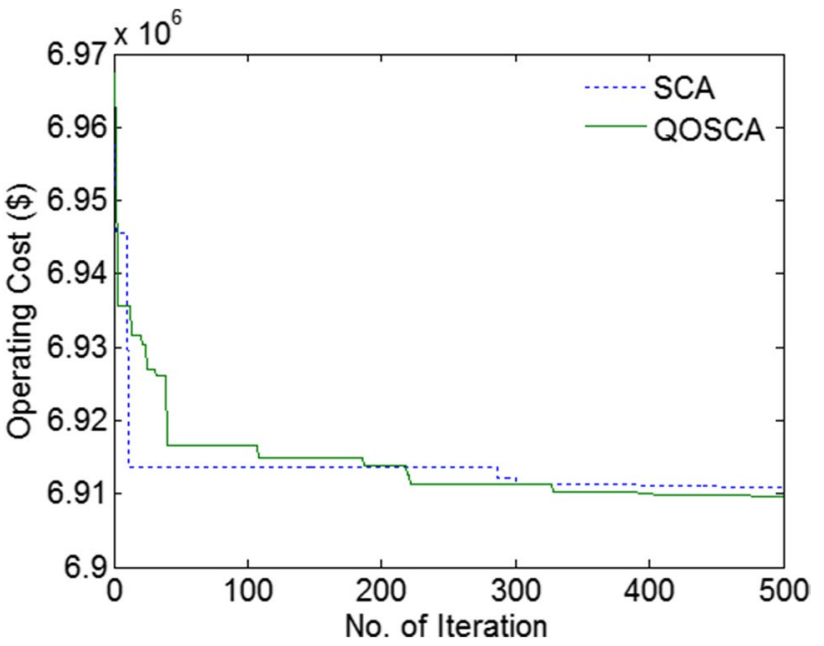

Fig. 5 Convergence curve of operating cost using SCA and QOSCA technique

candidly reveals the superiority of the proposed algorithm and the influence of QOSCA to avoid premature convergence and yield solutions with accuracy.

Similarly, In IEEE 30 bus test system, there are five generator buses with interconnected 41 transmission lines. Three branches are equipped with tap changing transformer and two shunt capacitors. Initially, active power loss without reactive power planning is $7.11 \mathrm{MW}$ and its operating cost is $3.737016 \times 10^{7} \$$. The search space of this test system has 13 dimension that includes five reactive power generators, three transformer tap settings and four shunt capacitors are placed at weak buses where a large amount of reactive power is present.

Table 5 represents the control parameters obtained by the proposed SCA. The results of proposed SCA are also compared with other optimization algorithm like Sens-GA [15], Sens-DE [15], Sens-PSO [15], SPSO [18], APSO

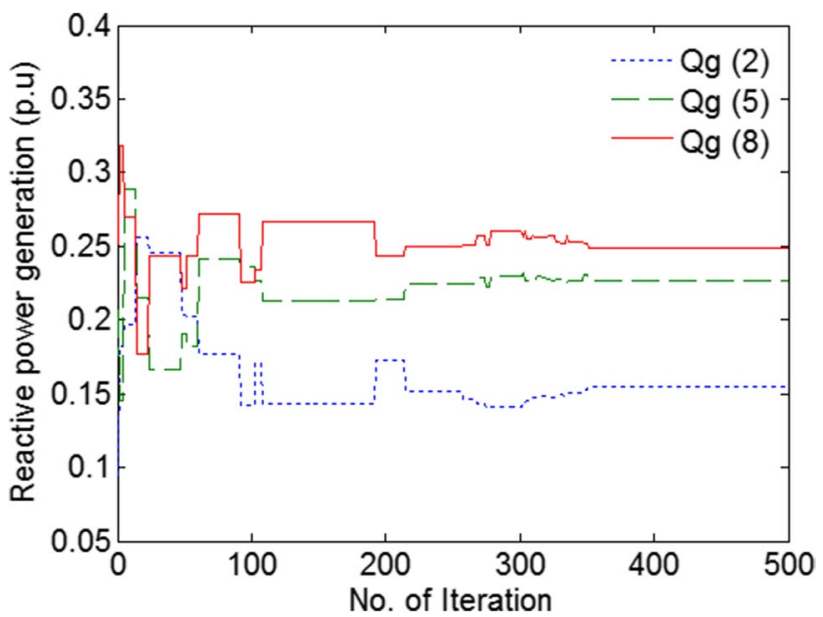

Fig. 6 Variation of Reactive power generation profile 


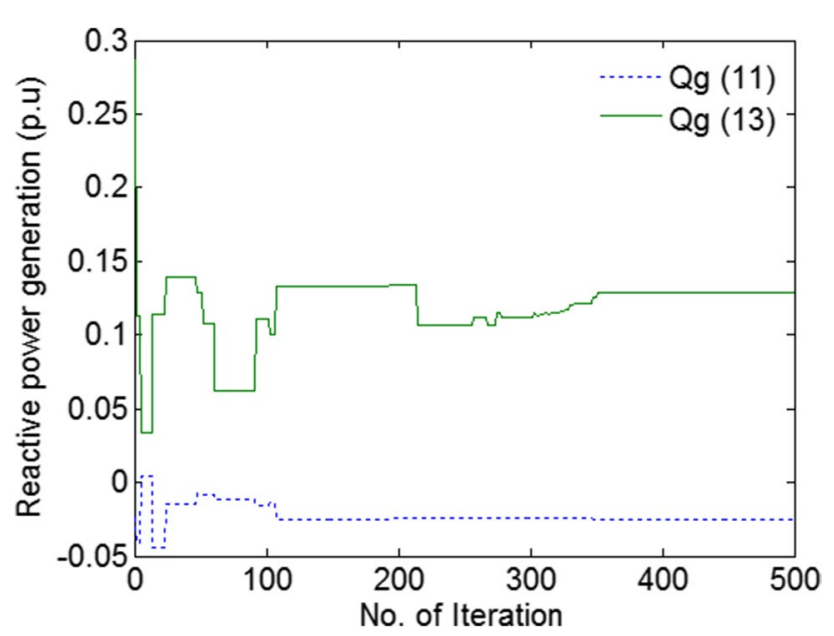

Fig. 7 Variation of Reactive power generation profile

Table 5 Optimal control parameters for minimum transmission loss and total operating cost when tested on the IEEE-57 bus system

\begin{tabular}{|c|c|c|}
\hline Variable & SCA & QOSCA \\
\hline \multicolumn{3}{|c|}{ Reactive generation of generators (in p.u) } \\
\hline $\mathrm{Q}_{\mathrm{G}}(2)$ & 0.1575 & 0.4942 \\
\hline $\mathrm{Q}_{\mathrm{G}}(3)$ & 0.4028 & 0.3094 \\
\hline $\mathrm{Q}_{\mathrm{G}}(6)$ & 0.0137 & 0.1013 \\
\hline $\mathrm{Q}_{\mathrm{G}}(8)$ & -0.4394 & 0.8443 \\
\hline $\mathrm{Q}_{\mathrm{G}}(9)$ & 0.0201 & 0.0599 \\
\hline $\mathrm{Q}_{\mathrm{G}}(12)$ & -0.0109 & 0.0759 \\
\hline \multicolumn{3}{|c|}{ Transformer tap positions (in p.u) } \\
\hline $\mathrm{T}(19)$ & 0.9114 & 0.9342 \\
\hline $\mathrm{T}(20)$ & 0.9123 & 0.9080 \\
\hline $\mathrm{T}(31)$ & 0.9958 & 0.9973 \\
\hline $\mathrm{T}(35)$ & 0.9152 & 0.9218 \\
\hline $\mathrm{T}(36)$ & 0.9000 & 0.9196 \\
\hline $\mathrm{T}(37)$ & 0.9979 & 0.9788 \\
\hline $\mathrm{T}(41)$ & 0.9000 & 0.9000 \\
\hline $\mathrm{T}(46)$ & 0.9000 & 0.9225 \\
\hline $\mathrm{T}(54)$ & 0.9016 & 0.9065 \\
\hline $\mathrm{T}(58)$ & 0.9021 & 0.9016 \\
\hline $\mathrm{T}(59)$ & 0.9348 & 0.9293 \\
\hline $\mathrm{T}(65)$ & 0.9044 & 0.9001 \\
\hline $\mathrm{T}(66)$ & 0.9000 & 0.9000 \\
\hline $\mathrm{T}(71)$ & 0.9000 & 0.9025 \\
\hline $\mathrm{T}(73)$ & 0.9980 & 0.9957 \\
\hline $\mathrm{T}(76)$ & 0.9721 & 0.9924 \\
\hline $\mathrm{T}(80)$ & 0.9028 & 0.9012 \\
\hline \multicolumn{3}{|l|}{ Shunt capacitors (in p.u) } \\
\hline $\mathrm{Q}_{\mathrm{C} 1}$ & 0.2971 & 0.2970 \\
\hline $\mathrm{Q}_{\mathrm{C} 2}$ & 0.2772 & 0.1573 \\
\hline $\mathrm{Q}_{\mathrm{C} 3}$ & 0.1393 & 0.2691 \\
\hline $\mathrm{Q}_{\mathrm{C} 4}$ & 0.1119 & 0.1800 \\
\hline Active power loss (p.u) & 0.2509 & 0.2482 \\
\hline Operating cost (\$) & $1.3188 \times 10^{7}$ & $1.3044 \times 10^{7}$ \\
\hline
\end{tabular}

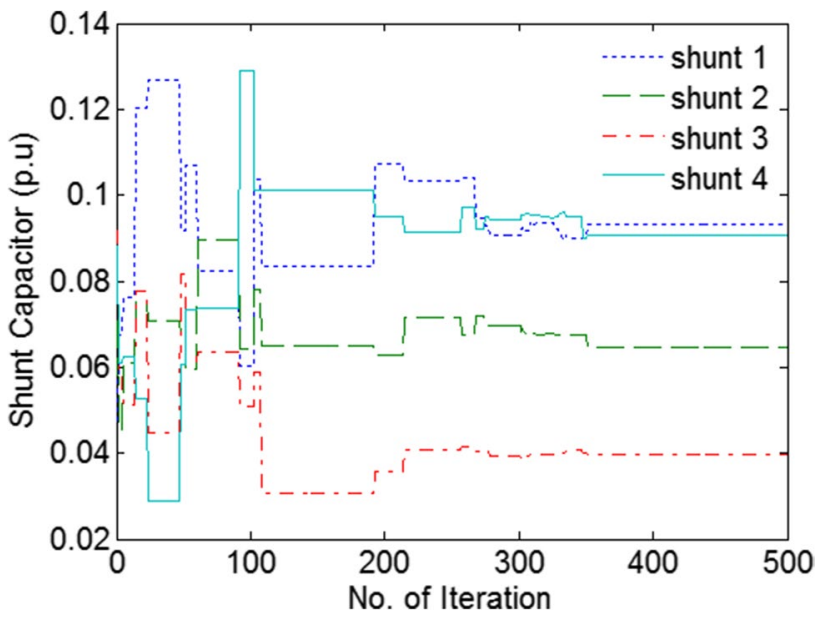

Fig. 8 Variation of Shunt capacitors for $\mathrm{Q}_{\mathrm{c}}(1), \mathrm{Q}_{\mathrm{c}}(2)$ and $\mathrm{Q}_{\mathrm{c}}(3)$

[18], EPSO [18], GWO [19] and OGWO [19]. With the application of the proposed approach, total active power loss is reduced to $0.0679 \mathrm{p} . \mathrm{u}$ and system operating cost is also reduced to $6.9097 \times 10^{6} \$$. Figures 6 and 7 depicts the reactive power generation variation at the generator buses during every iteration. Figures 8 and 9 displays the reactive power variation of shunt capacitors and transformer tap setting conditions respectively. The minimization of the active power loss curve is shown in Fig. 10 and the convergence characteristics curve of the system operating cost is shown in Fig. 11. From obtained tables and figures, it can be observed that the proposed QOSCA and SCA techniques are able to reduce real power loss to a large extent; hence, the capability of the transmission lines improves. It can also be observed that QOSCA

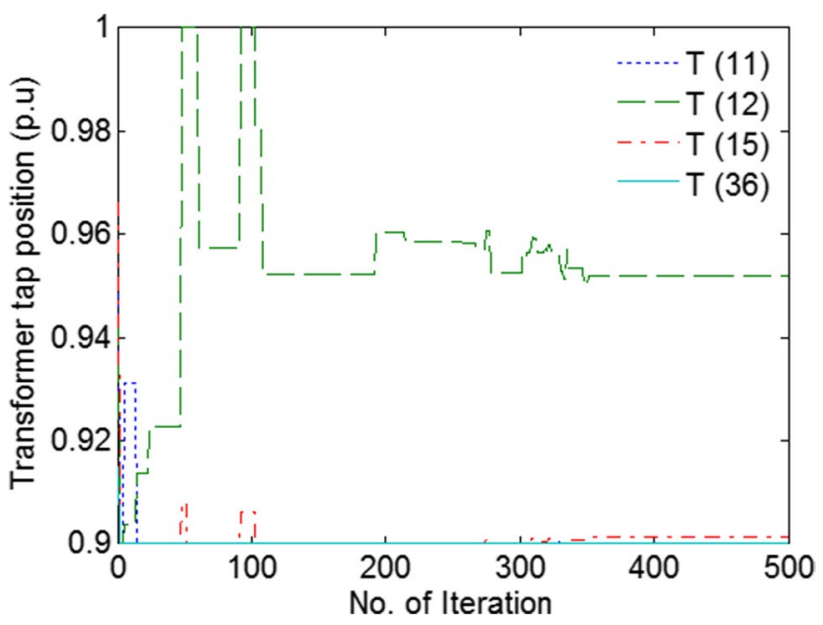

Fig. 9 Variation of Transformer Tap settings for T (6-9), T (6-10) and $\mathrm{T}(28-27)$ 


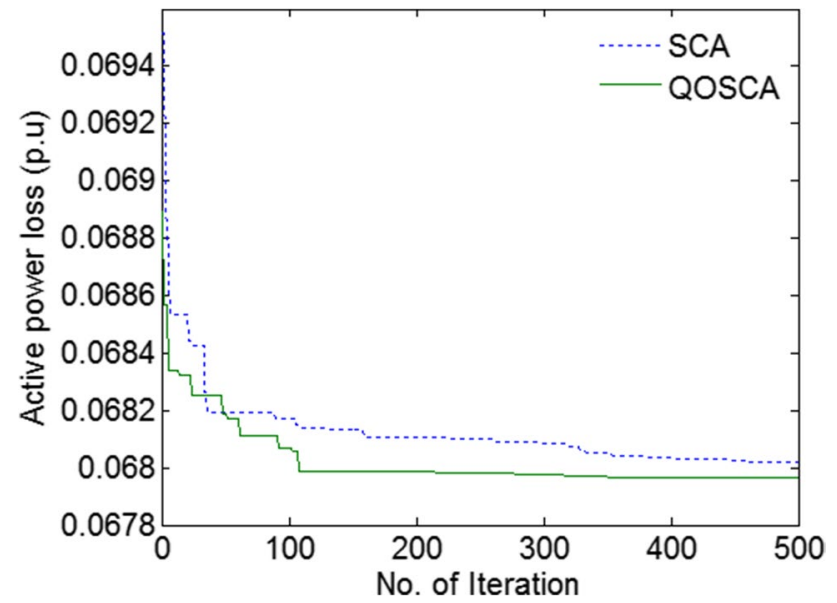

Fig. 10 Convergence curve of active power loss using SCA and QOSCA technique

optimization gives the better results as compared to the other optimization techniques.

The standard IEEE 57 bus test system consists of seven generating units at buses $1,2,3,6,8,9$ and 12 interconnected with 80 transmission lines and seventeen branches are equipped with tap changing transformer. In addition, there are shunt capacitors at buses 18, 25 and 53. Bus 1 is selected as slack bus. The total active power demand is $1251.70 \mathrm{MW}$ and reactive power demand is 335.70 MVAr at 100 MVA. Initially, active power loss without reactive power planning is $27.99 \mathrm{MW}$ and its operating cost is $1.471 \times 10^{7} \$$. The search space of this test system has 13 dimension that includes six reactive power generators, seventeen transformers tap settings and three shunt capacitors are placed at weak buses where

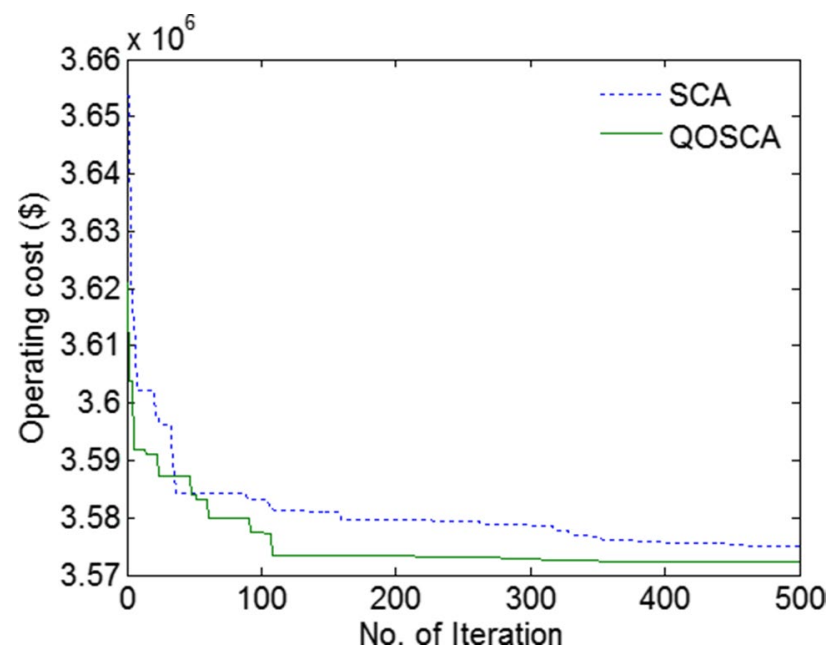

Fig. 11 Convergence curve of operating cost using SCA and QOSCA technique

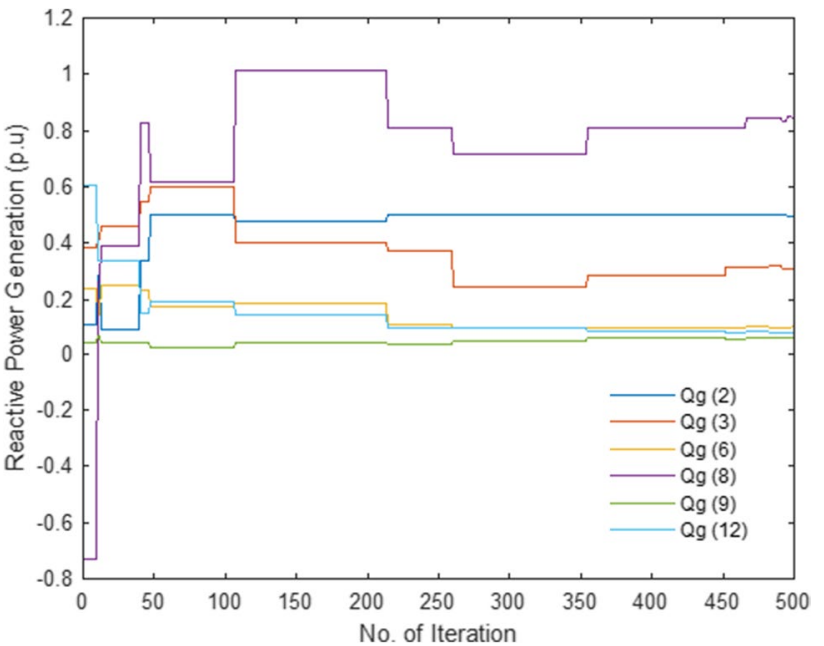

Fig. 12 Variation of Reactive power generation profile

a large amount of reactive power support is required. Table 5 shown best control parameter setting obtained by proposed SCA and QOSCA. With the application of the proposed approach, total active power loss is reduced to $0.2482 \mathrm{p}$.u and system operating cost is also reduced to $1.3044 \times 10^{7} \$$. Figure 12 depicts the reactive power generation variation at the generator buses during every iteration. Figures 13 and 14 displays the reactive power variation of shunt capacitors and transformer tap setting conditions respectively.

The convergence curve of active power loss using SCA and QOSCA technique is shown in Fig. 15. The convergence curve of operating cost using SCA and QOSCA technique is shown in Fig. 16.

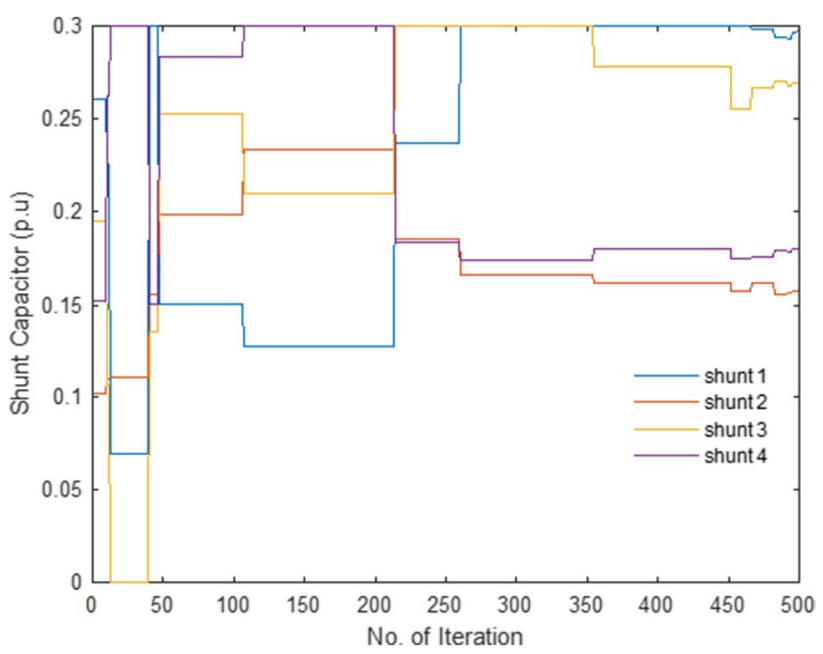

Fig. 13 Variation of Shunt capacitors for $\mathrm{Q}_{\mathrm{c}}(1), \mathrm{Q}_{\mathrm{c}}(2), \mathrm{Q}_{\mathrm{c}}(3)$ and $\mathrm{Q}_{\mathrm{c}}$ (4) 


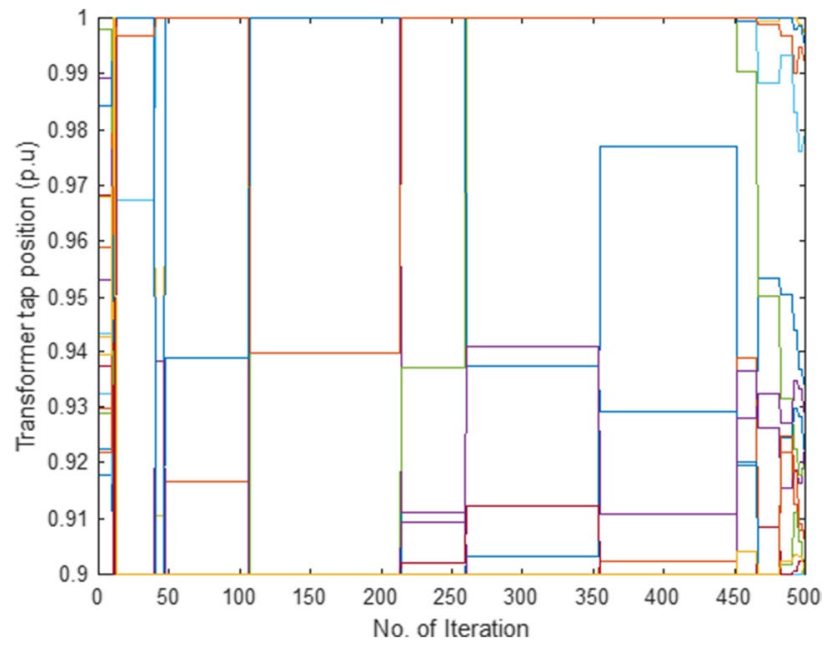

Fig. 14 Variation of Transformer Tap settings

\section{Conclusion}

Reactive power planning has been a crucial problem for power system operators. The prime importance of RPP is to minimize the active power losses and operating costs of the system. This planning is done through proper co-ordination of control variables, which are transformer tap setting arrangements, shunt capacitors and generation of reactive power by generators. Different state-of-the-art methods have been developed to reduce line losses in order to achieve an efficient and economical system. In this work, SSA and QOSSA techniques have been successfully implemented on standard IEEE 14 and IEEE 30 bus system of the connected power network. The proposed QOSCA reduces the

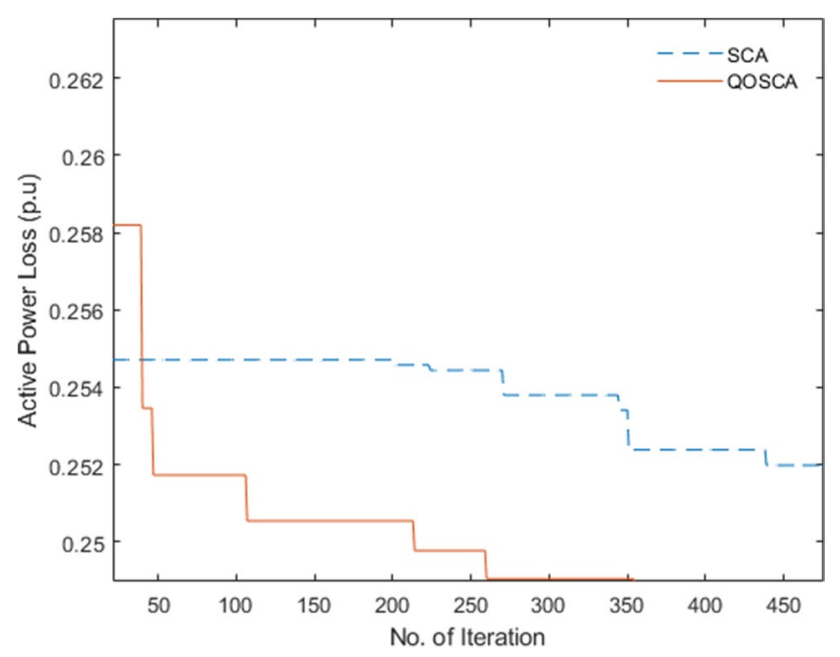

Fig. 15 Convergence curve of active power loss using SCA and QOSCA technique

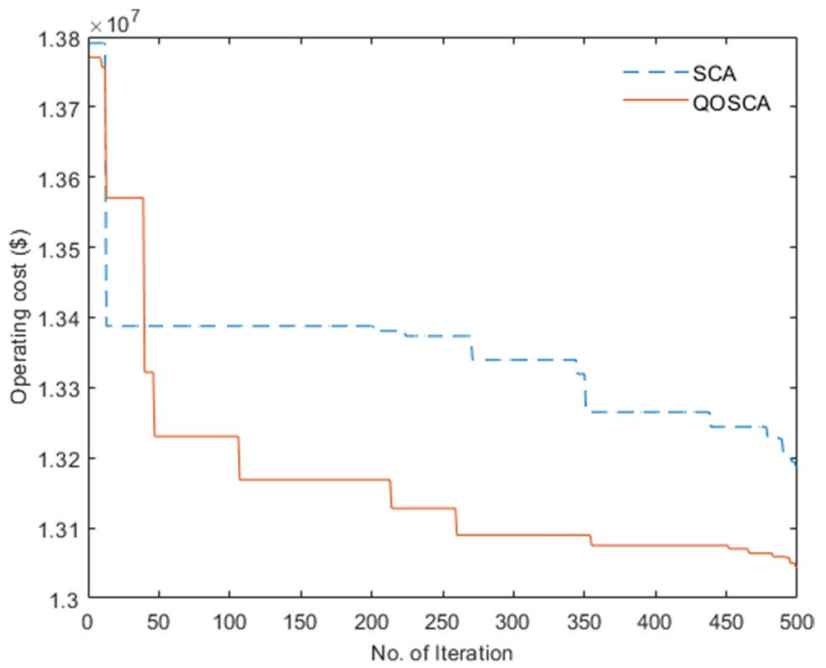

Fig. 16 Convergence curve of operating cost using SCA and QOSCA technique

transmission losses by $6.38 \%$ in IEEE $14,4.56 \%$ in IEEE 30 and $12.77 \%$ in IEEE 57 bus system. The outcomes are compared with other techniques used in the literature. It is observed that the QOSCA algorithm provides much better results than the other techniques used in recent state-of-literature. It can be concluded that the Sine cosine algorithm may be considered as an effective optimization technique for solving RPP problems and can be considered as a promising candidate for future researches.

\section{References}

1. Chiang HD, Wang JC, Cockings O, Shin HD (1990) Optimal capacitor placements in distribution systems. I. A new formulation and the overall problem. IEEE Trans Power Delivery 5(2):634-642

2. Chiang HD, Wang JC, Cockings O, Shin HD (1990) Optimal capacitor placements in distribution systems. II. Solution algorithms and numeric results. IEEE Trans Power Delivery 5(2):643-649

3. Lopez J, Pozo D, Contreras J, Mantovani JRS (2015) A convex chance-constrained model for reactive power planning. Int $\mathrm{J}$ Electr Power Energy Syst 71:403-411

4. Yousefi GR, Seifi H, Sepasian MS, Haghighat H et al (2004) A new reactive power planning procedure for Iranian Power Grid. Electric Power Systems Research 72(3):225-234

5. Mahmoudabadi A, Rashidinejad M (2013) An application of hybrid heuristic method to solve concurrent transmission network expansion and reactive power planning. Int J Electr Power Energy Syst 45(1):71-77

6. Sakr WS, EL-Sehiemy A Ragab, Azmy Ahmed M (2017) Adaptive differential evolution algorithm for efficient reactive power management. Applied Soft Computing 53:336-351

7. Rizk-Allah RM, El-Sehiemy RA, Wang GG (2018) A novel parallel hurricane optimization algorithm for secure emission/ economic load dispatch solution. Appl Soft Comput 63:206-222 
8. El-Fergany AA, Hasanien HM (2018) Tree-seed algorithm for solving optimal power flow problem in large-scale power systems incorporating validations and comparisons. Appl Soft Comput 64:307-316

9. Alamgir Hossain Md, Chakrabortty Ripon K, Ryan Michael J, Pota Hemanshu Roy (2021) Energy management of community energy storage in grid-connected microgrid under uncertain real-time prices. Sustainable Cities and Society 66:102658

10. López J, Contreras J, Mantovani JRS (2015) Reactive power planning under conditional-value-at-risk assessment using chance-constrained optimization. IET Gener Transm Distrib 9(3):231-240

11. Ugranli F, Karatepe E, Nielsen AH (2017) MILP Approach for Bilevel Transmission and Reactive Power Planning Considering Wind Curtailment. IEEE Trans Power Syst 32(1):652-661

12. Horestani ARK, Golshan MEH, Monsef H (2016) Expected security constrained reactive power planning. IET Gener Transm Distrib 10(10):2306-2315

13. Wang Y, Li F, Wan Q, Chen H (2011) Reactive Power Planning Based on Fuzzy Clustering, Gray Code, and Simulated Annealing. IEEE Trans Power Syst 26(4):2246-2255

14. Dai C, Chen W, Zhu Y, Zhang X (2009) Seeker optimization algorithm, for optimal reactive power dispatch. IEEE Transactions on Power System 24(3):1218-1231

15. Bhattacharyya B, Goswami SK, Bansal RC (2009) Loss Sensitivity Approach in Evolutionary algorithms for Reactive Power Planning. Electric Power Components \& Systems 37(3):287-299

16. Bhattacharyya B, Babu R (2016) Teaching learning based optimization algorithm for reactive power planning. Int J Electr Power Energy Syst 81:248-253

17. Shekarappa G, Swetha Sheila Mahapatra, Raj Saurav (2021) Voltage constrained reactive power planning problem for reactive loading variation using hybrid harris hawk particle swarm optimizer. Electric Power Components and Systems 49(4-5):421-435

18. Bhattacharyya B, Raj S (2016) PSO based bio inspired algorithms for reactive power planning. Int J Electr Power Energy Syst 74:396-402

19. Raj Saurav, Bhattacharyya Biplab (2018) Reactive power planning by opposition-based grey wolf optimization method. International Transactions on Electrical Energy Systems 28(6):e2551

20. Bhattacharyya B, Raj S (2017) Differential evolution technique for the optimization of reactive power reserves. Journal of Circuits, Systems and Computers 26(10)

21. Mirjalili S (2016) SCA: A Sine Cosine Algorithm for solving optimization problems. Knowl-Based Syst 96:120-133

22. Tizhoosh HR (2005) Opposition-based learning: a new scheme for machine intelligence. International Conference on Computational Intelligence for Modelling, Control and Automation and International Conference on Intelligent Agents, Web Technologies and Internet Commerce (CIMCA-IAWTIC'06) 1:695-701

23. Mahdad B (2019) Optimal reconfiguration and reactive power planning based fractal search algorithm: A case study of the Algerian distribution electrical system. An International Journal Engineering Science and Technology 22(1):78-101

24. Ettappan $\mathrm{M}$ et al (2020) Optimal reactive power dispatch for real power loss minimization and voltage stability enhancement using artificial bee colony algorithm. Microprocess Microsyst 103085
25. Mahapatra S, Dey B, Raj S (2021) A novel ameliorated Harris hawk optimizer for solving complex engineering optimization problems. Int J Intell Syst 36(12):7641-7681

26. Bhattacharyya B, Karmakar N (2020) A planning strategy for reactive power in power transmission network using soft computing techniques. Int J Power Energy Syst 40(3)141-148

27. Mahapatra S, Badi M, Raj S (2019) Implementation of PSO, it's variants and Hybrid GWO-PSO for improving Reactive Power Planning. 2019 Global Conference for Advancement in Technology (GCAT) 1-6. https://doi.org/10.1109/GCAT47503.2019. 8978348

28. G. Swetha Shekarappa, Mahapatra S, Raj S (2021) Voltage constrained reactive power planning by Ameliorated HHO Technique. In Recent Advances in Power Systems, Springer, Singapore, $p$ 435-443

29. Babu R, Raj S, Bhattacharyya B (2020) Weak bus-constrained PMU placement for complete observability of a connected power network considering voltage stability indices. Protection and Control of Modern Power Systems 5(1):1-14

30. Jabr RA, Martins N, Pal BC, Karaki S (2011) Contingency constrained $\mathrm{VAr}$ planning using penalty successive conic programming. IEEE Trans Power Syst 27(1):545-553

31. Pal Bijay Baran, Biswas Papun, Mukhopadhyay Anirban (2013) GA based FGP approach for optimal reactive power dispatch. Procedia Technology 10:464-473

32. Kumar SK Nandha, Renuga P (2009) Reactive power planning using differential evolution: comparison with real GA and evolutionary programming. International Journal of Recent Trends in Engineering 2(5): 130

33. Bhattacharyya B, Kumar S (2016) Loadability enhancement with FACTS devices using gravitational search algorithm. Int J Electr Power Energy Syst 78:470-479

34. Christy A Ananthi, Prashant Kumar (2012) Reactive power optimization based on PSO and considering voltage security in a practical power system. In 2012 International Conference on Emerging Trends in Science, Engineering and Technology (INCOSET), IEEE, $p$ 493-499

35. Lenin K, Ravindranath Reddy B, Surya Kalavathi M (2014) Water cycle algorithm for solving optimal reactive power dispatch problem. J Eng Technol Res 2(2):1-11

36. Ghasemi Mojtaba, Ghanbarian Mohammad Mehdi, Ghavidel Sahand, Rahmani Shima, Moghaddam EsmaeilMahboubi (2014) Modified teaching learning algorithm and double differential evolution algorithm for optimal reactive power dispatch problem: a comparative study. Information Sciences 278:231-249

37. Dutta Susanta, Paul Sourav, Roy Provas Kumar (2018) Optimal allocation of SVC and TCSC using quasi-oppositional chemical reaction optimization for solving multi-objective ORPD problem. Journal of Electrical Systems and Information Technology $5(1): 83-98$

Publisher's Note Springer Nature remains neutral with regard to jurisdictional claims in published maps and institutional affiliations. 